\title{
Use of artificial intelligence for public health surveillance: a case study to develop a machine Learning-algorithm to estimate the incidence of diabetes mellitus in France
}

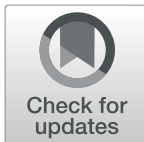

\author{
Romana Haneef ${ }^{1 *}$ (D, Sofiane Kab ${ }^{2 \dagger}$, Rok Hrzic ${ }^{3 \dagger}$, Sonsoles Fuentes ${ }^{1}$, Sandrine Fosse-Edorh ${ }^{1}$, \\ Emmanuel Cosson ${ }^{4,5}$ and Anne Gallay ${ }^{1}$
}

\begin{abstract}
Background: The use of machine learning techniques is increasing in healthcare which allows to estimate and predict health outcomes from large administrative data sets more efficiently. The main objective of this study was to develop a generic machine learning $(\mathrm{ML})$ algorithm to estimate the incidence of diabetes based on the number of reimbursements over the last 2 years.

Methods: We selected a final data set from a population-based epidemiological cohort (i.e., CONSTANCES) linked with French National Health Database (i.e., SNDS). To develop this algorithm, we adopted a supervised ML approach. Following steps were performed: i. selection of final data set, ii. target definition, iii. Coding variables for a given window of time, iv. split final data into training and test data sets, v. variables selection, vi. training model, vii. Validation of model with test data set and viii. Selection of the model. We used the area under the receiver operating characteristic curve (AUC) to select the best algorithm.

Results: The final data set used to develop the algorithm included 44,659 participants from CONSTANCES. Out of 3468 variables from SNDS linked to CONSTANCES cohort were coded, 23 variables were selected to train different algorithms. The final algorithm to estimate the incidence of diabetes was a Linear Discriminant Analysis model based on number of reimbursements of selected variables related to biological tests, drugs, medical acts and hospitalization without a procedure over the last 2 years. This algorithm has a sensitivity of $62 \%$, a specificity of $67 \%$ and an accuracy of $67 \%$ [95\% Cl: 0.66-0.68].
\end{abstract}

\footnotetext{
* Correspondence: Romana.HANEEF@santepubliquefrance.fr

' Sofiane Kab and Rok Hrzic contributed equally as a second author.

1 Department of Non-Communicable Diseases and Injuries, Santé Publique

France, 12 rue du Val d'Onse, 94415 Saint-Maurice, France

Full list of author information is available at the end of the article
}

(c) The Author(s). 2021 Open Access This article is licensed under a Creative Commons Attribution 4.0 International License, which permits use, sharing, adaptation, distribution and reproduction in any medium or format, as long as you give appropriate credit to the original author(s) and the source, provide a link to the Creative Commons licence, and indicate if changes were made. The images or other third party material in this article are included in the article's Creative Commons licence, unless indicated otherwise in a credit line to the material. If material is not included in the article's Creative Commons licence and your intended use is not permitted by statutory regulation or exceeds the permitted use, you will need to obtain permission directly from the copyright holder. To view a copy of this licence, visit http://creativecommons.org/licenses/by/4.0/. The Creative Commons Public Domain Dedication waiver (http://creativecommons.org/publicdomain/zero/1.0/) applies to the data made available in this article, unless otherwise stated in a credit line to the data. 
Conclusions: Supervised ML is an innovative tool for the development of new methods to exploit large health administrative databases. In context of InfAct project, we have developed and applied the first time a generic MLalgorithm to estimate the incidence of diabetes for public health surveillance. The ML-algorithm we have developed, has a moderate performance. The next step is to apply this algorithm on SNDS to estimate the incidence of type 2 diabetes cases. More research is needed to apply various MLTs to estimate the incidence of various health conditions.

Keywords: Artificial intelligence, Machine learning technique, Supervise learning, Health indicator, Incidence, Diabetes mellitus, Electronic health records and public health surveillance

\section{Background}

The availability of administrative data generated from different sources is increasing and the possibility to link these data sources with other databases offers unique opportunity to answer those research questions, which require a large sample size or detailed data on hard-toreach population [1]. French National Health Data System (i.e., SNDS [Système National de Données Santé]) is an example of a big data/large administrative linked data set, which is used for public health surveillance in France [2]. It includes most updated, individual level health information about health insurance claims, hospital discharge and mortality of whole French population (i.e., 66 million people) [2]. However, the estimation of health indicators from linked administrative data is challenging due to several reasons such as variability in data sources and data collection methods, availability of a large number of variables, lack of skills and capacity to analyze big data [3]. More efficient ways of analyzing health information using big data across European countries are required. In that context, the use of artificial intelligence (AI) is increasing in healthcare. Indeed AI allows to handle data with a large number of dimensions (features) and units (feature vectors) efficiently with a high precision. AI techniques offer benefits in estimation of health indicators both at individual and population levels (i.e., improving social and health policy process). Machine learning (ML) is an application of AI that provides systems the ability to learn automatically and improve from experience without being explicitly programmed [4]. Supervised learning algorithms build on a mathematical model of a set of data that contains both the inputs and the desired outputs [5]. This approach is based on the prior knowledge of what the output values for a given sample should be [6]. ML techniques have been applied for the diagnosis of certain conditions as well as outcome prediction and prognosis evaluation with high precision [7-9].

This study was carried out under the InfAct (Information for Action) project [10], which is a joint action of Member States aiming to develop a more sustainable European health information system through improving the availability of comparable, robust and policy-relevant health status data and health system performance information. InfAct gathers 40 national health authorities from 28 Member States. This study is part of a work package (WP9) focused on innovation in health information system (i.e., using data linkages and/or AI) to improve public health surveillance and health system performance for health policy process. As a first step, we have explored the current usage of these innovative techniques (i.e., data linkages and/or AI) in European countries and very few countries apply AI to estimate health indicators in their public health activities [11]. Therefore, the next step was to develop a generic approach by applying these innovative techniques to estimate the health indicators of chronic conditions for improved surveillance.

We used diabetes as a case study due to several reasons. First, it is one of the leading cause of morbidity in the world [12] and its prevalence is increasing among all ages in the European region, mostly due to increase in overweight and obesity, unhealthy diet and physical inactivity [13]. Second, a training data set using CONSTANCES cohort was already developed and used to answer various research questions for diabetes. Third, as this study is part of the InfAct project with a limited period to be completed. Fourth, estimation of incidence of diabetes cases is important to develop the prevention strategies to reduce its burden. For example, promoting healthy diet and physical activity in daily life could reduce the risk of developing diabetes 2 .

The main objective of this study was to develop for the first time a generic ML-algorithm to estimate the incidence of diabetes based on the number of reimbursements over the last 2 years, excluding the anti-diabetes drugs as predictors and focused on non-diabetic participants over the last 2 years.

\section{Method}

\section{Development of the ML-algorithm}

To develop ML-algorithm, we adopted a supervised ML approach using R-software $(\mathrm{R} \times 643.6 .3)$ and used following key libraries: caret 6.0-86, AppliedPredictiveModeling 1.1-7, CORElearn 1.54.2, C50 0.1.3.1, and xgboost 1.3.2.1. Following steps were performed: i. selection of final data set, ii. target definition, iii. Coding of 
variables for a given window of time, iv. split final data into training and test data sets, v. variables selection, vi. training model, vii. Validation of model with test data set and viii. Selection of the model.

\section{i. Selection of final data set}

We selected a final data set from a population-based epidemiological cohort (i.e., CONSTANCES) to develop an algorithm to estimate the incidence of diabetes. The participants were recruited by CONSTANCES between January 1, 2012, and December 31, 2014. This cohort comprises after final completion a national representative randomly selected sample of 50,954 aged between 18 and 69 years (inclusive) and living in France [14, 15]. The participants are randomly selected from the beneficiaries of the National Health Insurance Fund (i.e. CNAM [Caisse Nationale d'Assurance Maladie]). In this cohort, data are collected using a self-administered questionnaire (SAQ) and a medical questionnaire (MQ) and were used to define the known diabetes cases and pharmacologically-treated diabetes [16]. For known diabetes cases, in the SAQ, participants reported to have diabetes through the item: "Have you ever been told by a doctor or other health care professional that you had diabetes?" In the medical questionnaire, completed during the medical examination, the physician asked each participant if they had diabetes. For the pharmacologicallytreated diabetes, two questions in the medical questionnaire were related to diabetes treatment: "Are you currently being treated for diabetes with oral medication?" And "Are you currently being treated for diabetes with one or more insulin injections?" [16].

After fulfilling a SAQ on health status, life style factors, socioeconomic and demographic characteristics, the participants attend to their related health screening center for a medical examination which includes: medical questionnaire, physical examination and blood sampling. This information previously collected was linked with the French National Health Data System (i.e., SNDS). We excluded women who declared being already diagnosed of gestational diabetes mellitus, pregnant women, no data on participants in SNDS, incomplete data in SAQ/MQ, incomplete data on age of diabetes diagnosis, diabetes cases who were declared before 12 months of SAQ/MQ and all participants with antidiabetic drug reimbursement between 12 and 36 months before SAQ/MQ. Moreover, we considered gestational diabetes as a special group and excluded these women due to their different physiopathology. Some may develop the diabetes earlier or later due to hormonal disturbance. To predict the incidence of diabetes among these women required specific case definitions.

\section{ii. Target definition}

The target definition includes the participants who declared diabetes in CONSTANCES cohort (first occurrence $\leq 12$ months) with the first antidiabetic drug reimbursement between 0 and 12 months before inclusion. The diabetes status at inclusion (M0: inclusion in CONSTANCES) was defined according to CONSTANCES as described above. The linkage with the French National Health Data System (i.e., SNDS) allowed recording all antidiabetic drug reimbursement between 0 and 36 months before inclusion (SAQ/MQ).

Participants without declared diabetes (CONSTANCES SAQ/MQ) and/or antidiabetic drug reimbursement between 0 and 36 months before inclusion were defined as non-diabetes cases (target 0 ). Participants who declared diabetes in CONSTANCES SAQ/ MQ (first occurrence $\leq 12$ months) with the first antidiabetic drug reimbursement $\leq 12$ months before inclusion were defined as incident cases (target 1). These diabetes cases included both type 1 and 2. Participants with antidiabetic drug reimbursement between 12 and 36 months before inclusion or declared diabetes (first occurrence $>$ 12 months) were excluded (see Fig. 1). We excluded these participants to avoid the potential influence of anti-diabetes drugs on the estimation of incidence of diabetes (not true incident cases).

\section{iii. Coding of variables for a given window of time}

In CONSTANCES, we only coded those variables, which were also available in the SNDS to apply the potential ML-algorithm on SNDS to estimate the incidence of diabetes. A total of 3483 continuous variables were coded and standardized using the z-score transformation (for each data point, we subtracted the mean and divided with the standard deviation) over the last 24 months before the date of SAQ. The rational to have a time window of 24 months before the SAQ was to provide a long duration to study changes in diagnostic procedures, hospitalizations and drug consumption that allows to estimate the incidence of diabetes with high accuracy. Following were the main categories of variables: number of medical consultations (50 variables), drug dispensed coded using the 5th level of the Anatomical Therapeutic code [ATC 05] (461 variables), biological test (747 variables), medical acts (i.e., X-ray, surgery, etc.) (2135 variables), all hospitalizations (5 variables), hospitalizations with a procedure (i.e., dialysis, radiotherapy, etc.) (5 variables), hospitalizations without a procedure (5 variables), hospitalizations related to following associated health conditions: diabetes, heart failure, stroke, heart attack, foot ulcer, lower limb amputation, ischemic heart disease, transient ischemic attack, end-stage renal failure, diabetic coma, diabetic ketoacidosis and cancer (75 variables). 


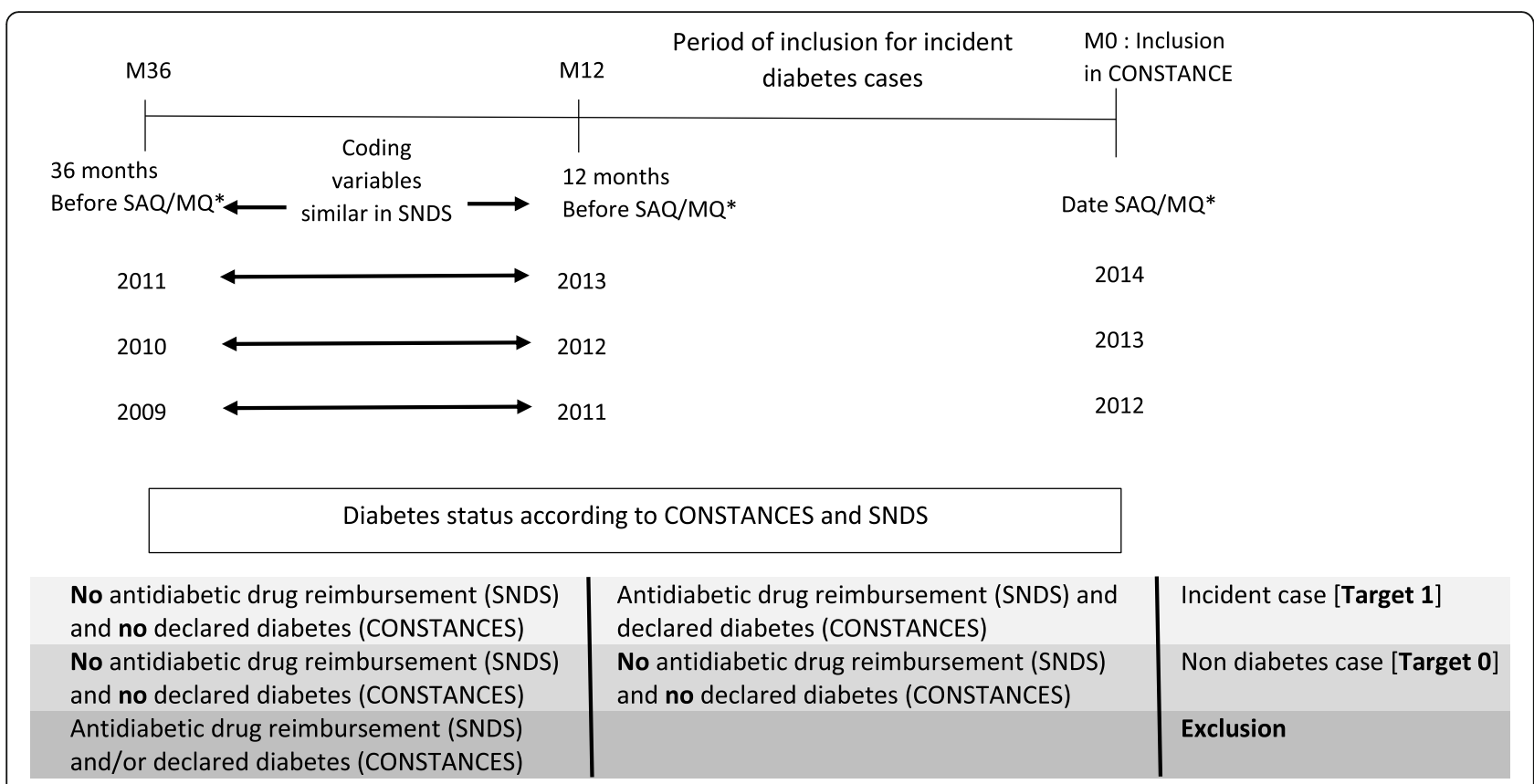

Fig. 1 Target definition in CONSTANCES Cohort, "A case study performed in 2019-20 to develop a Machine Learning-algorithm to estimate the incidence of Diabetes Mellitus in France". *SAQ: Self-administered Questionnaire. MQ: Medical Questionnaire

\section{iv. Split final data set into training and test data sets}

The final data set was randomly split into $80 \%$ as a training data set and $20 \%$ as test data set. There was a significant imbalance of number of positive target (i.e., target $1=$ diabetes treated cases) over the number of negative target (i.e., target $0=$ non-diabetes cases) in the training dataset. To avoid the bias in ML-algorithm and skew in class distribution, we performed a random down sampling in the training data set in target 0 group to achieve the same number of individuals in both target groups. This includes 35,728 participants where target 0 includes 35,663 participants and target 1 includes 65. Random down sampling was performed on target 0 until to achieve the same number of target 1 i.e., 65 .

The selection of variables and the model was performed using the training data. The test data was used solely to test the final model performance.

\section{v. Variables selection}

First, we removed all variables with a variance equal to zero and then the ReliefF exp. score was estimated, based on the relevance of each variable, to differentiate between target 1 and target 0 . The ReliefF expRank method is noise tolerant and is not affected by features interactions [17-19]. All the variables were ranked according to the ReliefF exp. score. For continuous variables, the score values range from 0 to 1 [18]. The cutoff score was 0.01 and was selected based on the visual inspection of the ordered plot of ReliefF values for all variables, called "elbow plot" approach. The variables that had a ReliefF exp. score equal or more than 0.01 were included to train different models and the variables less than 0.01 were excluded.

\section{Steps vi to viii model selection and validation of the model with test data set}

The four following models [i.e., 1. Linear discriminant analysis (LDA), 2. Logistic regression (LR), 3. Flexible discriminant analysis (FDA) and 4. Decision tree model (C5)] were applied to the training data set. We also fit three boosted algorithms (1. Boosted logistic regression, 2. Boosted C5, and 3. XGBoost), to test whether these more computationally intensive algorithms can perform significantly better than the more standard four models. The AUC is the most commonly used evaluation/performance metric in machine learning studies and measure the ability of a classifier to distinguish between classes. We used the area under the receiver operating characteristic curve (AUC) in both the cross-validation steps and the best (final) algorithm. For each model, we compared the performance in terms of AUC. We used five-fold cross-validation repeated three times to fit each model in the training stage. After that, the models' performances were assessed using the testing data set. We calculated the mean distributions of variables to highlight each predictor, the relative difference in the two predicted groups (diabetic, non-diabetic), for example the mean of age was $56.70 \mathrm{y}$ for the predicted diabetics and $43.69 \mathrm{y}$ for non-diabetics. 


\section{Sensitivity analysis}

Unbalanced training data set can skew the class distribution that may affect the performance of the machine learning algorithm. To address this issue, random resampling was applied to balance the training data set and to avoid the bias estimation. We applied two following techniques: 1 . over sampling is the random repeated sampling from the minority class (positive target: diabetes cases) that artificially inflates its prevalence and 2. Down sampling is the random repeated sampling from the majority class (negative target: non-diabetes cases) that artificially reduces its prevalence. The sensitivity analysis was performed for over and down sampling approaches on the test data set. Then, we automated the model selection process by giving the computer a specific metric including sensitivity, specificity, positive predictive value, negative predictive value and kappa. Finally, a single model was retained based on its performance and its transferability to other databases.

\section{Results}

\section{Final data set}

The final data set to develop the algorithm included 44,659 participants, with 81 incident diabetes cases (target 1) and 44,578 participants without diabetes (target 0) (Fig. 2). The general characteristics of the final data set is described in Table 1. The incident diabetes group was included older, with a higher percentage of men, treated hypertension and dyslipidemia, former smokers, a higher body mass index and a family history of diagnosed diabetes as compared to non-diabetes group.

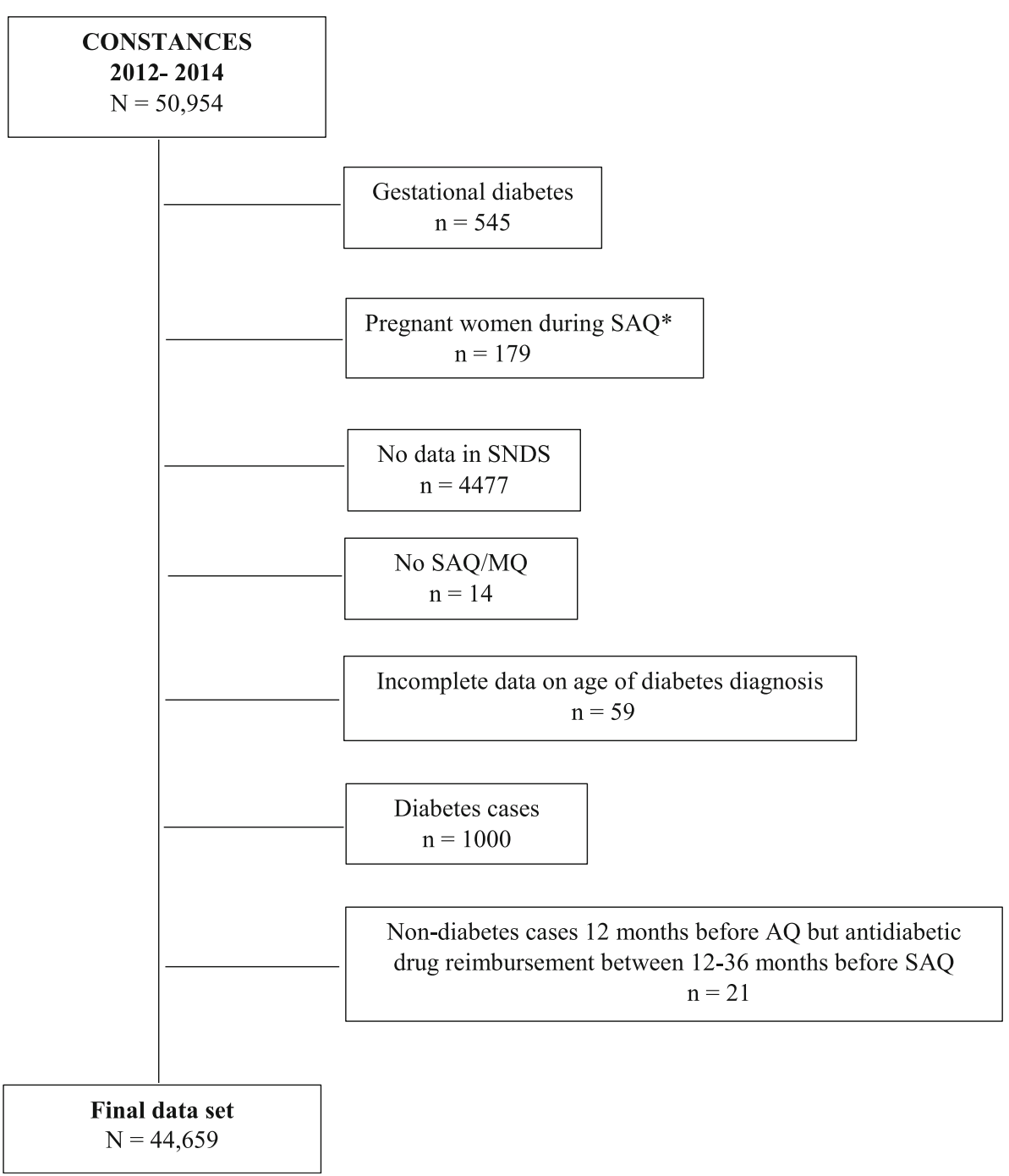

Fig. 2 Flow chart for the selection of the final data set from CONSTANCES Cohort, "A case study performed in 2019-20 to develop a Machine Learning-algorithm to estimate the incidence of Diabetes Mellitus in France". ${ }^{*} A Q=$ Self-administered Questionnaire. MQ = Medical Questionnaire 
Table 1 General characteristics of final data set (i.e., study population). "A case study performed in 2019-20 to develop a Machine Learning-algorithm to estimate the incidence of Diabetes Mellitus in France"

\begin{tabular}{|c|c|c|c|}
\hline \multirow[t]{2}{*}{ Study Population } & \multirow{2}{*}{$\begin{array}{l}\text { Total } \\
(N=44,659)\end{array}$} & \multicolumn{2}{|l|}{$N=44,659$} \\
\hline & & $\begin{array}{l}\text { Target } 0 \text { (Non-diabetes } \\
\text { cases }=44,578)\end{array}$ & $\begin{array}{l}\text { Target } 1 \text { (Incident diabetes } \\
\text { cases }=81 \text { ) }\end{array}$ \\
\hline Age in years, mean $( \pm S D)$ & $47.8, \pm 13.2$ & $47.8, \pm 13.2$ & $57.0, \pm 8.2$ \\
\hline Gender, men \% (n) & 46.9 (20946) & 46.9 (20896) & $61.7(50)$ \\
\hline \multicolumn{4}{|l|}{ Smoking status, \% (n) } \\
\hline Never smoked & $43.2(19296)$ & $43.2(19271)$ & $30.9(25)$ \\
\hline Former smoker & $33.1(14772)$ & $33.1(14741)$ & $38.3(31)$ \\
\hline Current smoker & $18.6(8320)$ & $18.6(8307)$ & $16.0(13)$ \\
\hline Missing & $5.1(2271)$ & $5.1(2259)$ & $14.8(12)$ \\
\hline Body mass index, $\mathrm{kg} / \mathrm{m}^{2}$ (mean, $\pm \mathrm{SD}$ ), (n) & $25.0, \pm 4.4(43668)$ & 25.0, $\pm 4.4(43588)$ & $31.8, \pm 6.0(80)$ \\
\hline Treated hypertension, yes, \% (n) & $11.3(5031)$ & $11.2(4996)$ & $43.2(35)$ \\
\hline Treated dyslipidemia, yes, \% (n) & $8.1(3635)$ & $8.1(3609)$ & $32.1(26)$ \\
\hline Mother/father diagnosed with diabetes, yes, \% (n) & $15.1(6764)$ & $15.1(6730)$ & $42.0(34)$ \\
\hline \multicolumn{4}{|l|}{ Education ${ }^{\mathrm{a}} \%(\mathrm{n})$} \\
\hline No education - primary education & $3.1(1374)$ & $3.1(1366)$ & $9.9(8)$ \\
\hline Lower secondary education & $6.9(3060)$ & $6.8(3042)$ & $22.2(18)$ \\
\hline Upper secondary education & 33.5 (14942) & $33.4(14911)$ & $38.3(31)$ \\
\hline Lower tertiary education & $33.0(14728)$ & $33.0(14714)$ & $17.3(14)$ \\
\hline Upper tertiary education & 21.7 (9709) & $21.8(9699)$ & $12.3(10)$ \\
\hline Missing or other category & $1.9(846)$ & $1.9(846)$ & $0()$. \\
\hline \multicolumn{4}{|l|}{ Geographical origin, \% (n) } \\
\hline Metropolitan France & 87.9 (39249) & $87.9(39177)$ & $88.9(72)$ \\
\hline $\mathrm{FOT}^{\mathrm{b}}$ & $0.9(381)$ & $0.9(379)$ & $2.5(2)$ \\
\hline Europe & $4.2(1861)$ & $4.2(1859)$ & $2.5(2)$ \\
\hline North Africa & $2.8(1260)$ & $2.8(1257)$ & $3.7(3)$ \\
\hline Sub-Saharan Africa & $1.1(503)$ & $1.1(502)$ & $1.2(1)$ \\
\hline Asia & $0.7(326)$ & $0.7(326)$ &.$()$. \\
\hline Others & $1.0(433)$ & $1.0(433)$ &.$()$. \\
\hline Missing or don't want to answer & $1.4(646)$ & $1.4(645)$ & $1.2(1)$ \\
\hline \multicolumn{4}{|l|}{ Professional activity, \% (n) } \\
\hline Employed & $65.2(29123)$ & $65.3(29093)$ & $37.0(30)$ \\
\hline Unemployed & $6.1(2721)$ & $6.1(2712)$ & $11.1(9)$ \\
\hline Retired & $21.8(9753)$ & $21.8(9720)$ & 40.7 (33) \\
\hline Student & $1.5(653)$ & $1.5(653)$ & $0()$. \\
\hline Unemployed due to disability & $0.9(390)$ & $0.9(385)$ & $6.2(5)$ \\
\hline No professional activity & $1.4(603)$ & $1.4(602)$ & $1.2(1)$ \\
\hline Missing or other category & $3.2(1416)$ & $3.2(1413)$ & $3.7(3)$ \\
\hline
\end{tabular}

SD Standard Deviation

a Based on the International Classification ISCED

${ }^{b}$ French overseas territories

\section{Variables selection}

Out of 3468 continuous variables coded, 23 variables $(0.7 \%)$ had a ReliefF exp. Score above 0.01, ranked based on this score and were therefore selected (Fig. 3) (Table 2).
The first variable was the "age". The following nine were related to "number of reimbursements of biological tests performed in last 2 years" (i.e., Alkaline Phosphatase test, Gamma Glutamyle Transferase test, Transaminases (ALAT and ASAT, TGP and TGO) blood test, 


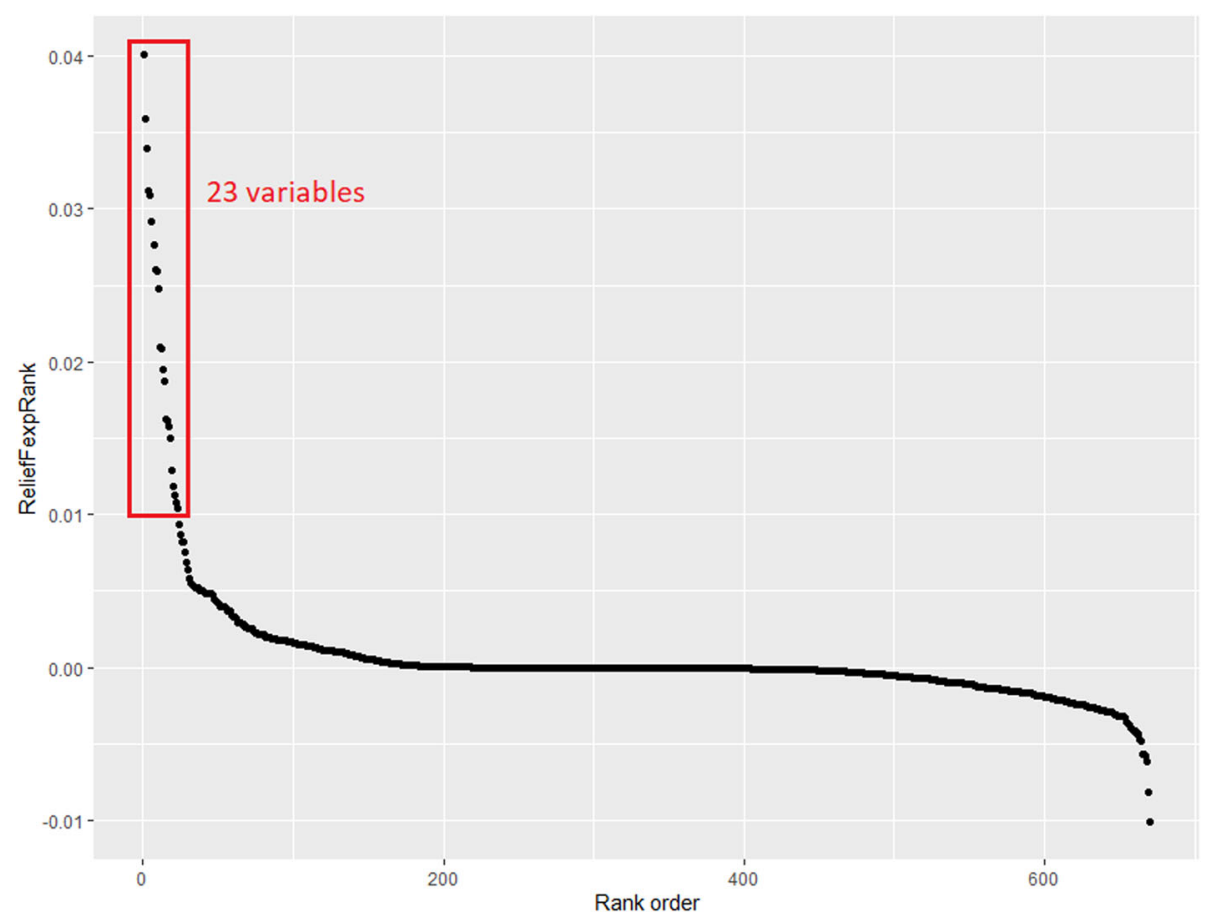

Fig. 3 Variables selection based on ReliefF Exp Score, "A case study performed in 2019-20 to develop a Machine Learning-algorithm to estimate the incidence of Diabetes Mellitus in France"

Uric Acid (Uricemia) blood test, glucose blood, Creatinine level blood test, Exploration of a Lipid Anomaly (ELA) blood test, HbA1c test and C-Reactive Protein test). The next seven were related to "number of reimbursements of various non-diabetes drugs in last 2 years" (i.e., Proton pump inhibitors drugs, antidiarrheal drugs, Penicillin with broad spectrum drugs, bacterial and viral vaccines, Acetic acid derivatives, Propionic acid derivatives and Anilides (Paracetamol). The following five were related to "number of reimbursements of various medical acts" (i.e., fundus examination by biomicroscopy with contact lens, functional examination of ocular motricity, binocular vision examination, mammography and X-ray for thorax). The last one is "the total number of hospitalizations without a procedure (i.e., dialysis, chemotherapy) in last 2 years".

\section{Algorithm to estimate the incidence of diabetes}

After the selection of variables, four different models [i.e., 1. Linear discriminant analysis (LDA), 2. Logistic regression (LR), 3. Flexible discriminant analysis (FDA) and 4. Decision tree model (C5)], were trained with the training dataset using three repeats of five-fold crossvalidation graph. The performance of each of the model was tested on the test dataset and was measured by AUC, for which an empirical 95\% confidence interval was calculated based on 15 resamples with replacement (Fig. 4). After that, we compared the performances of these four models using test data set to select the one based on the performance metrics (Table 3). We kept the LDA model since it showed a better performance with an accuracy of $67 \%$ with the test data set as compared to other models (Table 3). The three boosted algorithms improved on the predictive performance as compared to the standard models (Fig. 4). The accuracy of boosted version of logistic regression, the boosted C5.0 classification model, and XGBoost was 77, 67 and $69 \%$, respectively (see Additional file 1). The results of sensitivity analysis are reported in the Additional file 1.

\section{Distribution of means of selected variables in test data set}

After the selection of LDA model, the 23 selected variables were trained with the test data set $(20 \%$ of final data set $44,659=8931)$. We compared the distribution of means of these continuous variables among two groups: incident diabetes cases (i.e., 2921) and non- diabetes incident cases (i.e., 6010) using LDA algorithm in the test data set (Table 4). The 2921 diabetes patients in the test dataset are the predicted cases. The mean distribution of all selected variables related to the number of reimbursements of biological tests, medicines not used for diabetes treatment and medical acts performed in last 2 years, was significantly higher in the incident diabetes group than in non-diabetes group. For example, the age was the first ranked variable with 0.04 ReliefF exp. score 
Table 2 List of selected variables ranked based on their ReliefF Exp Score. "A case study performed in 2019-20 to develop a Machine Learning-algorithm to estimate the incidence of Diabetes Mellitus in France"

\begin{tabular}{|c|c|c|}
\hline Ranked \# & CATEGORIES & Independent Variables \\
\hline 1 & AGE & Age in years \\
\hline \multicolumn{3}{|c|}{ Diabetes related variables } \\
\hline 6 & BIOLOGICAL TESTS & $\mathrm{Nb}$. of reimbursement of Glucose blood test in last 2 years \\
\hline 9 & BIOLOGICAL TESTS & $\mathrm{Nb}$. of reimbursement of $\mathrm{HbA} 1 \mathrm{c}$ tests in last 2 years \\
\hline 18 & MEDICAL ACTS & $\mathrm{Nb}$. of reimbursement of Fundus examination by biomicroscopy with contact lens in last 2 years \\
\hline 19 & MEDICAL ACTS & $\mathrm{Nb}$. of reimbursement of functional examination of the ocular motricity in last 2 years \\
\hline 20 & MEDICAL ACTS & $\mathrm{Nb}$. of reimbursement of binocular vision examination in last 2 years \\
\hline \multicolumn{3}{|c|}{ Non-diabetes related variables } \\
\hline 2 & BIOLOGICAL TESTS & $\mathrm{Nb}$. of reimbursement of Alkaline Phosphatase test in last 2 years \\
\hline 3 & BIOLOGICAL TESTS & Nb. of reimbursement of Gamma Glutamyle Transferase test in last 2 years \\
\hline 4 & BIOLOGICAL TESTS & Nb. of reimbursement of Transaminases (ALAT and ASAT, TGP and TGO) blood test in last 2 years \\
\hline 5 & BIOLOGICAL TESTS & $\mathrm{Nb}$. of reimbursement of Uric Acid (Uricemia) blood test in last 2 years \\
\hline 7 & BIOLOGICAL TESTS & $\mathrm{Nb}$. of reimbursement of Creatinine level blood test in last 2 years \\
\hline 8 & BIOLOGICAL TESTS & $\mathrm{Nb}$. of reimbursement of Exploration of a Lipid Anomaly (ELA) blood test in last 2 years \\
\hline 10 & BIOLOGICAL TESTS & $\mathrm{Nb}$. of reimbursement of C-Reactive Protein test in last 2 years \\
\hline 11 & DRUGS & $\mathrm{Nb}$. of reimbursement of Proton pump inhibitors drugs in last 2 years \\
\hline 12 & DRUGS & $\mathrm{Nb}$. of reimbursement of other antidiarrheal drugs in last 2 years \\
\hline 13 & DRUGS & $\mathrm{Nb}$. of reimbursement of Penicillin with broad spectrum drugs in last 2 years \\
\hline 14 & DRUGS & $\begin{array}{l}\text { Nb. of reimbursement of bacterial and viral vaccines, combined (diphtheria-haemophilus influenza } \\
\text { B-pertussis-tetanus-hepatitis B-meningococcal A }+C \text { ) in last } 2 \text { years }\end{array}$ \\
\hline 15 & DRUGS & $\mathrm{Nb}$. of reimbursement of Acetic acid derivatives and related substances in last 2 years \\
\hline 16 & DRUGS & $\mathrm{Nb}$. of reimbursement of Propionic acid derivatives in last 2 years \\
\hline 17 & DRUGS & Nb. of reimbursement of Anilides (Paracetamol) in last 2 years \\
\hline 21 & MEDICAL ACTS & $\mathrm{Nb}$. of reimbursement of mammography, in last 2 years \\
\hline 22 & MEDICAL ACTS & $\mathrm{Nb}$. of reimbursement of $\mathrm{X}$-ray thorax in the previous 2 years in last 2 years \\
\hline 23 & HOSPITALIZATION & Total number of hospitalizations without a procedure (i.e. dialysis, chemotherapy) in last 2 years \\
\hline
\end{tabular}

among 23 selected variables and was highly discriminant in the incident diabetes group. The mean age of patients in diabetes group was 57 years old as compared to 44 years old in non-diabetes group (Table 4).

Following the age variable, nine other features selected, related to the mean number of reimbursements of biological tests, were more discriminant (i.e., the behavior of distinguishing features or characteristics of variables by comparing two groups) in incident diabetes group than in non-diabetes group. These biological tests were performed to measure the normal values of certain enzymes, proteins, glucose and uric acid in the blood to check the normal functions of liver, kidney, pancreas and other organs. For example, the mean number of reimbursement of blood glucose test in last 2 years was 1.74 times higher in diabetes predicted group than in non-diabetes predicted group. The following group of features was the mean number of reimbursements of drugs. There were seven drugs and their mean number of reimbursements in last 2 years was more discriminant in incident diabetes group than in non-diabetes group. In the category of medical acts, there were three following features more discriminant in incident diabetes group: mean number of reimbursements of examination of fundus by biomicroscopy with contact lens, ocular motricity and binocular vision in last 2 years.

There were seven unusual features selected by the ML-algorithm and were discriminant in incident diabetes group: mean number of reimbursements of broadspectrum penicillin, vaccines, propionic acid, Anilides (Paracetamol), mammography, X-ray for thorax and mean number of hospitalizations without any procedure.

\section{Discussion}

We have developed an algorithm based on the supervised ML approach to estimate the incidence of diabetes using a training data set from a cohort study. This algorithm (i.e., LDA model) was built on 23 selected variables from the CONSTANCES based on the number of reimbursements over the last 2 years to estimate the 


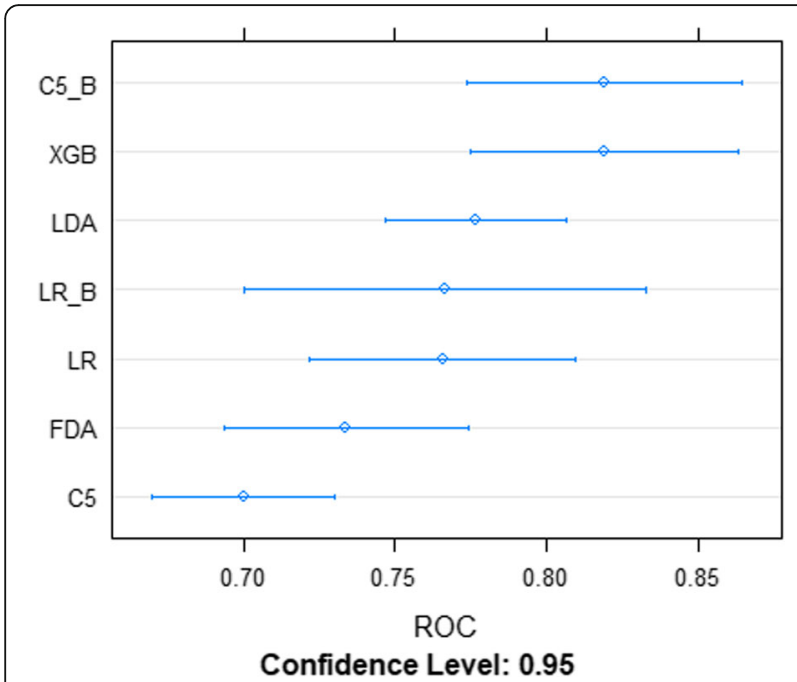

Fig. 4 Area under the receiver operating characteristic curve and the empirical 95\% confidence interval (based on fifteen resamples with replacement) for all models using the test dataset, "A case study performed in 2019-20 to develop a Machine Learningalgorithm to estimate the incidence of Diabetes Mellitus in France"

incidence of diabetes. This algorithm showed a moderate performance in predicting the incidence of diabetes cases with a sensitivity of $62 \%$ and an accuracy of $67 \%$. Among 23 selected variables, six were related to diabetes that were expected, such as age and Glucose blood test. Whereas 17 other variables were not directly related to diabetes and were more discriminant in incident diabetes group than in non-diabetes group such as Proton pump inhibitors drug.

Table 3 Model performance evaluation with test data set, "A case study performed in 2019-20 to develop a Machine Learning-algorithm to estimate the incidence of Diabetes Mellitus in France"

\begin{tabular}{lllll}
\hline & LDA & LR & FDA & C5 \\
\hline Accuracy & 0,67 & 0,65 & 0,66 & 0,64 \\
95\% Cl: & $(0,66-0,68)$ & $(0,64-0,66)$ & $(0,65-0,67)$ & $(0,63-0,65)$ \\
No Information Rate: & 0,998 & 0,998 & 0,998 & 0,998 \\
P-Value [Acc > NIR]: & 1000 & 1000 & 1000 & 1000 \\
Kappa & 0,003 & 0,004 & 0,002 & 0,003 \\
McNemar's Test P-Value & $<2 \mathrm{e}-16$ & $<2 \mathrm{e}-16$ & $<2 \mathrm{e}-16$ & $<2 \mathrm{e}-16$ \\
Sensitivity & 0,625 & 0,750 & 0,563 & 0,625 \\
Specificity & 0,673 & 0,650 & 0,661 & 0,640 \\
Pos Pred Value & 0,003 & 0,004 & 0,003 & 0,003 \\
Neg Pred Value & 0,999 & 0,999 & 0,999 & 0,999 \\
F1-statistics & 2,50 & 3,0 & 2252 & 2,50 \\
Detection Rate & 0,001 & 0,001 & 0,001 & 0,001 \\
Balanced Accuracy & 0,649 & 0,700 & 0,612 & 0,633 \\
\hline
\end{tabular}

\section{Main limitations of the ML-algorithm}

This study was performed as a proof of concept using ML-approach to estimate the incidence of diabetes cases from reimbursement data. The results have highlighted low accuracy and there are several aspects that could explain this low accuracy. Here are some limitations: first, small number of diabetes-treated cases in the final data set, which could be related to the lack of older population in CONSTANCES cohort (participants between 18 and 69 years at inclusion), maybe potentially linked with low accuracy. Participation in a cohort like CONSTANCES is cumbersome and demands additional time to take part in health examinations. People in less good health and having co-morbidities (including both old and young people), require regular health check-ups therefore, they could less motivation to participate in cohort studies. Thus, it required to wait few years of follow-up for volunteers to include older age groups to have more incident diabetes cases. The risk of developing diabetes increases with age, therefore by including larger number of older people in the final dataset, the performance of this algorithm may be improved. Second, the time window used to code the variables was previous 2 years, was long duration. We included a longer window to better evaluate the changes in diagnostic procedures, number of hospitalizations and drug consumption and to estimate the incidence of diabetes with high accuracy. More research is needed to explore different time windows and their impact on accuracy level of estimates. Third is related to diabetes, which is a complex medical condition with two major clinical types of diabetes, type 1 diabetes and type 2 diabetes. The pathology and dynamics of developing these two types of diabetes are very different. The type 1 diabetes is thought to be due to autoimmunological destruction of the Langerhans Islets hosting pancreatic- $\beta$-cells. It is diagnosed at very early stage of life and is believed to involve a combination of genetic and environmental factors. Whereas the main causes of type 2 diabetes are due to lifestyle, physical activity, dietary habits and genetic, is usually developed at later than 50 years of life. In our study, we defined the pharmacologically treated diabetes cases as target 1 and non-diabetes cases as target 0 . However, we did not explicitly define the pharmacological treated diabetes cases to be further characterized as type 1 and type 2. With the inclusion of this information in the model, the accuracy level of the model could be enhanced. Fourth, the predictors from reimbursement data may not have a strong ability to predict the diabetes outcome. Fifth, these results highlight the low level of model specificity. Considering the low incidence of diabetes, it is important to take into account that insufficient model specificity could lead to the overestimation of the diabetes incidence. These results should be interpreted with 


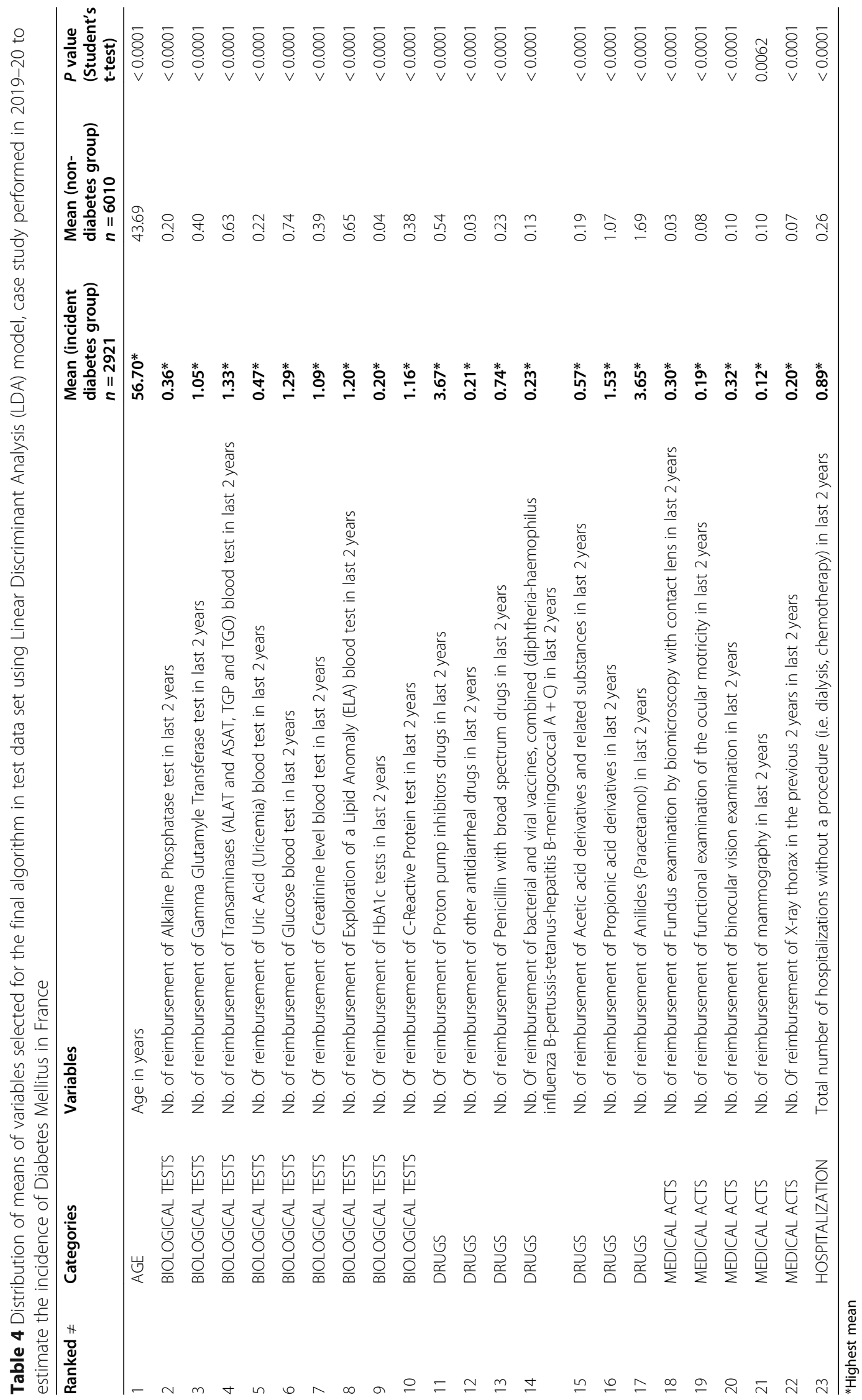


caution. Sixth, we applied an automated procedure and selected 23 the most related variables to our outcome based on a ReliefF exp. score equal or more than 0.01 . We did not consult is with a clinician. We recommend to combining both upstream clinical expertise and automated approaches for future research, for example by defining more complex indicators (more specific algorithms) related to specific conditions with higher risk of developing diabetes such as hypertension or dyslipidaemia, this can improve the performance of the algorithms. Finally, using the boosted algorithm for over sampling requires a high computational capacity and may take several days to compute the results. The computational capacity that is currently routinely available to public health institutions limits the attractiveness of complex analytical models that, while potentially highly accurate, may require several days to fit. This often results in such models being the product of specific research projects instead of being routinely developed in-house to support population health monitoring and clinical decisionmaking. This unfortunately partially limits the access to the advantages of advanced analytics. We therefore recommend that public health institutions invest in highperformance computing capacity.

Despite these limitations of this ML-algorithm, this study has some strengths: first is using supervised machine learning approach, we have developed the innovative methodology and could be applied to address other research questions. Second, this approach allows to reduce the dimension of a large number of variables (i.e., 3468) and identifies the most relevant variables (i.e., 23/ $3468=0.7 \%)$ to the desired outcomes more efficiently. The LDA model has been used for variables selection and dimensionality reduction for diabetes diagnosis [20]. Third, it allows to identify new variables, which were not observed using classical statistical approaches and can enrich the information to estimate the health indicators.

Our study has highlighted that among the 23 selected variables in the final model, five could be related to diabetes "number of Glucose blood test", "number of reimbursement of HbA1c tests", "number of Fundus examination by biomicroscopy with contact lens", "number of reimbursement of functional examination of the ocular motricity" and "number of reimbursement of binocular vision examination". These tests/examinations could happen after a diagnosed diabetes (without treatment in our study) or also characterize a group with higher risk factors but with not diagnosed diabetes. Further, three variables among the five are not specific to diabetes ("number of Fundus examination by biomicroscopy with contact lens", "number of reimbursement of functional examination of the ocular motricity" and "number of reimbursements of binocular vision examination. We also performed an additional analysis after implementing the model without these 5 variables, the accuracy was $65 \%$ (81\% sensitivity and $65 \%$ specificity). In France, the screening recommendations for diabetes are based on the glucose blood test. HbAlc is only recommended for the management of diabetes but not for diagnoses. In 2009 and 2010, the WHO has introduced $\mathrm{HbA1c}$ as an alternative method to diagnose diabetes that has been adopted by many countries since this date. The ophthalmologic problems such as glaucoma, cataract, ocular movement disorders, etc., are the main complications of diabetes. Therefore, the increase frequency of medical acts performed as a result of diabetes related complications such as visual functions allowed to better characterize incident diabetes cases. Moreover, the increased use of non-diabetic drugs along with mentioned biological tests in incident diabetes group may explain potentially the pre-existing comorbidity or may be late diagnosis diabetes with cardiovascular or gastrointestinal diseases. The aim was to estimate the incidence of treated diabetes, therefore all participants with prior antidiabetic drugs (12-36 months before inclusion) were excluded and therefore the antidiabetic drugs were not included among 23 selected variables. The main reason to exclude these participants were to capture the true estimation of incidence of diabetes and took into account other variables to predict the incidence of diabetes.

\section{Implications and perspectives for future research}

This innovative approach has been applied to two further studies: i. to classify and to estimate the prevalence of type 1 and type 2 diabetes cases [21] and, ii. to identify the number of undiagnosed diabetes cases ML algorithms in the SNDS (on going). For the first study, MLalgorithm developed has a sensitivity of $100 \%$ and specificity of $97 \%$, and for the second study, the sensitivity is $71 \%$ and specificity is $61 \%$.

The next step is to apply this algorithm on SNDS to estimate the incidence of type 2 diabetes cases. We recommend further research for following perspectives using ML-techniques: first to use different time windows (for example 6 months, 12 months or 16 months) to code variables and to explore their impact on estimates, sec$\underline{\text { ond }}$ to predict the trend of diabetes over time and third, to estimate the contribution of determinants of diabetes such as BMI, dietary habits and physical activity, on developing type 2 diabetes using ML approaches.

\section{Conclusions}

The use of MLT to analyze large administrative databases (health and non-health related data sources) is increasing across European countries in order to improve the public health surveillance and health policy process. Supervised machine learning is an innovative methodology for the development of algorithms to exploit large 
health administrative databases. It was the first step that we have developed a generic ML-algorithm with a moderate performance to estimate the incidence of diabetes in a training data set. The results of this study have highlighted important methodological steps to apply MLTs and their implications on large health administrative databases. The next step is to apply this algorithm on SNDS to estimate the incidence of type 2 diabetes cases. More research is needed to apply various MLTs to estimate the incidence of various health conditions and to calculate the contribution of various risk factors on developing type 2 diabetes.

\section{Abbreviations}

ML: Machine Learning; SNDS: Système National de Données Santé: French National Health Database; CONSTANCES: A population-based epidemiological cohort; Al: Artificial Intelligence; InfAct: Information for Action i.e., a joint action of Member States to establish a sustainable European health information system; WP: Work Package; CNAMTS: Caisse Nationale de I'Assurance Maladies des Travailleurs Salaries; SAQ: Self-administered Questionnaire; MQ: Medical Questionnaire; ATC: Anatomical Therapeutic Code; LDA: Linear Discriminant Analysis; LR: Logistic Regression; FDA: Flexible Discriminant Analysis; C5: Decision Tree; AUC: Area under Receiver Operating Characteristics Curve; Pos Pred Value: Positive Predictive Value; Neg Pred Value: Negative Predictive Value; 95\%Cl: 95\% Confidence Interval; BMI: Body Mass Index; WHO: World Health Organization

\section{Supplementary Information}

The online version contains supplementary material available at https://doi. org/10.1186/s13690-021-00687-0.

Additional file 1. It describes the summary of results with three boosted algorithms [boosted version of logistic regression, boosted C5.0 classification model, and XGBoost] to our set of four models [i.e., 1. Linear discriminant analysis (LDA), 2. Logistic regression (LR), 3. Flexible discriminant analysis (FDA) and 4. Decision tree model (C5)]. It includes Table S 1.1 (main analysis), Table S1.2 (sensitivity analysis: over sampling), Table S1.3 (sensitivity analysis: down sampling), Table S2 (description of models used and the set of hyperparameters explored) and Table S3 (Area under curve: AUC).

\section{Acknowledgements}

We acknowledge Le Marie Zins (Responsible for CONSTANCES cohort) for her kind support to access and use the data from this cohort.

\section{Authors' contributions}

Conceived and designed the study: RHaneef SFuentes RHrzic AG. Performed the study: RHaneef SKab RHrzic SFuentes. Analyzed the data: RHaneef SKab RHrzic SFuentes. Interpretation of the results: All authors contribute to the interpretation of the results. Contributed to the writing of the manuscript: All authors contributed to the writing of the manuscript. All authors read and approved the final manuscript.

\section{Funding}

This research has been carried out in the context of the project '801553 / InfAct' which has received funding from the European Union's Health Programme (2014-2020).

\section{Availability of data and materials}

Not applicable.

\section{Declarations}

Ethics approval and consent to participate Not applicable.

\section{Consent for publication}

All authors gave the consent for publication.

\section{Competing interests}

R. Haneef is the first author of this paper and the section editor of "health information system" of "Archives of Public Health". All other authors declare that they have no competing interests related to the work.

\section{Author details}

'Department of Non-Communicable Diseases and Injuries, Santé Publique France, 12 rue du Val d'Onse, 94415 Saint-Maurice, France. ${ }^{2}$ Population-Based Epidemiological Cohorts Unit, INSERM UMS 011, Villejuif, France.

${ }^{3}$ Department of International Health, Care and Public Health Research Institute - CAPHRI, University of Maastricht University, Maastricht, The Netherlands. ${ }^{4}$ Department of Endocrinology-Diabetology-Nutrition, AP-HP, Avicenne Hospital, Paris 13 University, Sorbonne Paris Cité, CRNH-IdF, CINFO, Bobigny, France. ${ }^{5}$ Sorbonne Paris Cité, UMR U1153 Inserm/U1125 Inra/Cnam/ Université Paris 13, Bobigny, France.

Received: 25 December 2020 Accepted: 2 September 2021

Published online: 22 September 2021

\section{References}

1. Harron K, Dibben C, Boyd J, Hjern A, Azimaee M, Barreto ML, et al. Challenges in administrative data linkage for research. Big Data Soc. 2017; 4(2):2053951717745678. https://doi.org/10.1177/2053951717745678.

2. Tuppin PRJ, Constantinou P, et al. Value of a national administrative database to guide public decisions: from the. Rev Epidemiol Sante Publique. 2017;65(4):S149-67. https://doi.org/10.1016/j.respe.2017.05.004.

3. Bradley CJ, Penberthy L, Devers KJ, Holden DJ. Health Services Research and Data Linkages: Issues, Methods, and Directions for the Future. Health Serv Res. 2010;45(5p2):1468-88.

4. Machine Learning: https://www.expertsystem.com/machine-learningdefinition/. 2017.

5. Russell S, Norvig P: Artificial Intelligence: A Modern Approach: https:// repository.unimal.ac.id/1022/1/Artificial\%20Intelligence\%20-\%20A\%2 OModern\%20Approach\%203rd\%20Ed\%20-\%20Stuart\%20Russell\%20and\%2 OPeter\%20Norvig\%2C\%20Berkeley\%20\%282010\%29.pdf. University Text Book (Third Edition) 2009.

6. Soni D: Supervised vs Unsupervised Learning: https://towardsdatascience. com/supervised-vs-unsupervised-learning-14f68e32ea8d. 2018.

7. Jha S, Topol EJ. Adapting to artificial intelligence: radiologists and pathologists as information specialists. JAMA. 2016;316(22):2353-4. https:// doi.org/10.1001/jama.2016.17438.

8. Patel VL, Shortliffe EH, Stefanelli M, Szolovits $P$, Berthold MR, Bellazzi $R$, et al. The coming of age of artificial intelligence in medicine. Artif Intell Med. 2009:46(1):5-17. https://doi.org/10.1016/j.artmed.2008.07.017.

9. Kavakiotis I, Tsave O, Salifoglou A, Maglaveras N, Vlahavas I, Chouvarda I. Machine Learning and data mining methods in diabetes research. Comput Struct Biotechnol J. 2017;15:104-16. https://doi.org/10.1016/j.csbj.2016.12. 005.

10. Joint Action on Health Information: https://www.inf-act.eu/. 2018.

11. Haneef $R$, Delnord M, Vernay M, Bauchet E, Gaidelyte R, Van Oyen $H$, et al. Innovative use of data sources: a cross-sectional study of data linkage and artificial intelligence practices across European countries. Arch Public Health. 2020;78(1):55. https://doi.org/10.1186/s13690-020-00436-9.

12. Cho NH, Shaw JE, Karuranga S, Huang Y, da Rocha Fernandes JD, Ohlrogge AW, et al. IDF diabetes atlas: global estimates of diabetes prevalence for 2017 and projections for 2045. Diabetes Res Clin Pract. 2018;138:271-81. https://doi.org/10.1016/j.diabres.2018.02.023.

13. WHO-Europe: The challenges of diabetes: http://www.euro.who.int/en/hea Ith-topics/noncommunicable-diseases/diabetes/data-and-statistics.

14. CONSTANCES: http://www.constances.fr/index_EN.php\#assets. 2019

15. Zins M. Goldberg M, team C: the French CONSTANCES population-based cohort: design, inclusion and follow-up. Eur J Epidemiol. 2015;30(12):131728. https://doi.org/10.1007/s10654-015-0096-4.

16. Fuentes $\mathrm{S}$, Cosson E, Mandereau-Bruno L, Fagot-Campagna A, Bernillon P, Goldberg M, et al. Identifying diabetes cases in health administrative databases: a validation study based on a large French cohort. Int Jo Public Health. 2019;64(3):441-50. https://doi.org/10.1007/s00038-018-1186-3. 
17. Chaix B, Kestens Y, Bean K, Leal C, Karusisi N, Meghiref K, et al. Cohort profile: residential and non-residential environments, individual activity spaces and cardiovascular risk factors and diseases--the RECORD cohort study. Int J Epidemiol. 2012;41(5):1283-92. https://doi.org/10.1093/ije/dyr107.

18. Kononenko MR-Sl: An adaption of Relief for attribute estimation in regression: http://www.clopinet.com/isabelle/Projects/reading/robnik97-icml. pdf. 1997.

19. Devaney M, Ram A. Machine Learning: proceedings of the fourteenth international conference, Nashville, TN, July 1997 (to appear); 2004.

20. Çalişir D, Doğantekin E. An automatic diabetes diagnosis system based on LDA-wavelet support vector machine classifier. Expert Syst Appl. 2011;38(7): 8311-5. https://doi.org/10.1016/j.eswa.2011.01.017.

21. Fuentes S, Hrzic R, Haneef R, Kab S, Fosse-Edorh S, Cosson E. Development of type 1/type 2 classification algorithm through machine learning methods and its application to surveillance using a nationwide database in France in: Diabetologia; 2020

\section{Publisher's Note}

Springer Nature remains neutral with regard to jurisdictional claims in published maps and institutional affiliations.

Ready to submit your research? Choose BMC and benefit from:

- fast, convenient online submission

- thorough peer review by experienced researchers in your field

- rapid publication on acceptance

- support for research data, including large and complex data types

- gold Open Access which fosters wider collaboration and increased citations

- maximum visibility for your research: over $100 \mathrm{M}$ website views per year

At BMC, research is always in progress.

Learn more biomedcentral.com/submissions 